\title{
Le mythe et le réenchantement de l'écriture, Albert Camus et Romain Gary en quête d'une parole originelle
}

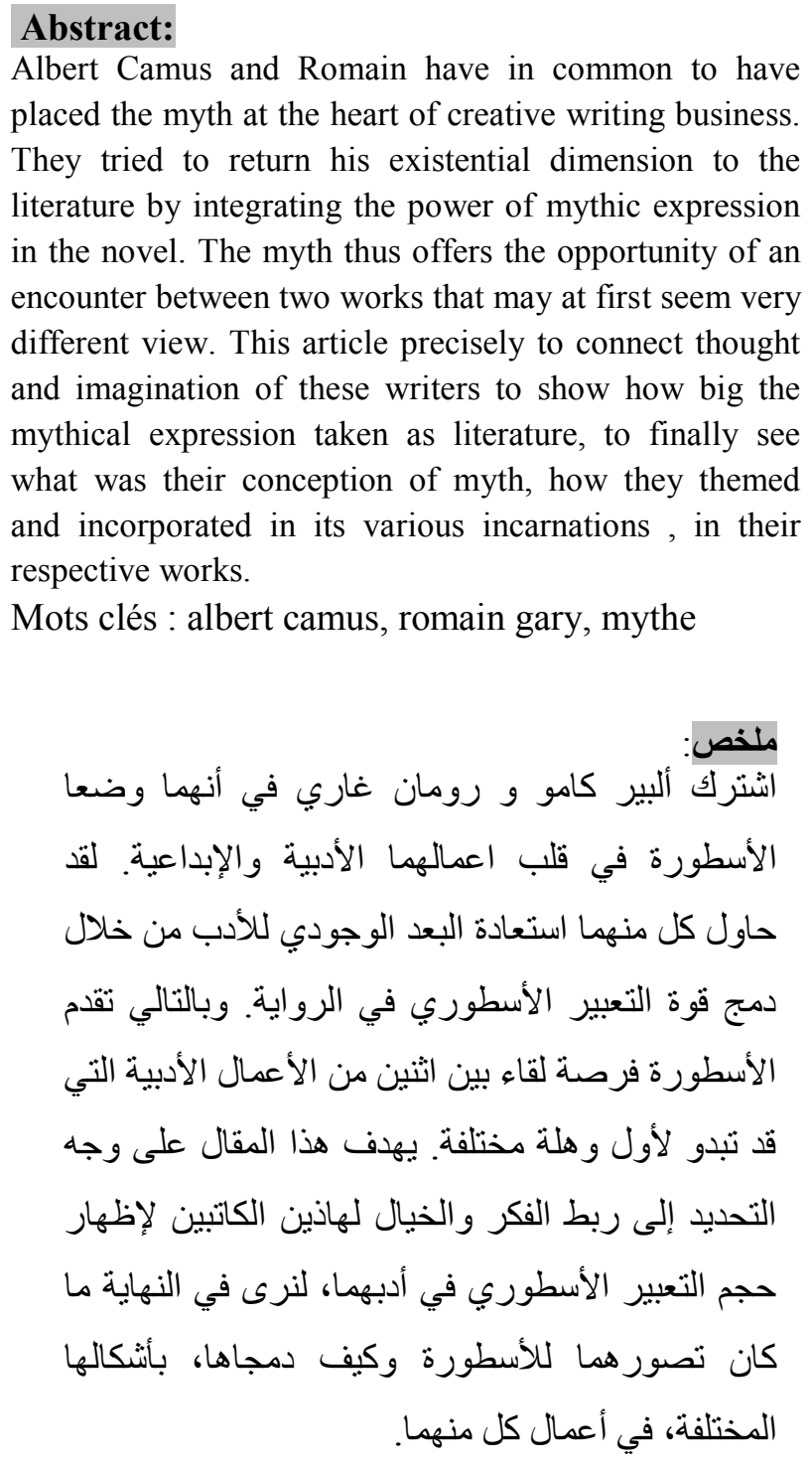

Albert Camus and Romain have in common to have placed the myth at the heart of creative writing business. They tried to return his existential dimension to the literature by integrating the power of mythic expression in the novel. The myth thus offers the opportunity of an encounter between two works that may at first seem very different view. This article precisely to connect thought and imagination of these writers to show how big the mythical expression taken as literature, to finally see what was their conception of myth, how they themed and incorporated in its various incarnations, in their respective works.

Mots clés : albert camus, romain gary, mythe

$$
\begin{aligned}
& \text { ملخص: أشترك ألبير كامو و رومان غاري في أنهما وضعا } \\
& \text { الأسطورة في قلب اعمالهما الأدبية والإبداعية. لقد } \\
& \text { حاول كل منهما استعادة البعد الوجودي للأدب من خلال } \\
& \text { دمج قوة التعبير الأسطوري في الرواية. وبالتالي تقدم } \\
& \text { الأسطورة فرصة لقاء بين اثثين من الأعمال الأدبية التي } \\
& \text { قد تبدو لأول وهلة مختلفة. يهدف هذا المقال على وجه } \\
& \text { التحديد إلى ربط الفكر و الخيال لهاذين الكاتبين لإظهار } \\
& \text { حجم التعبير الأسطوري في أدبهما، لنرى في النهاية ما } \\
& \text { كان تصور هما للأسطورة وكيف دمجاها، بأثكالها } \\
& \text { المختلفة، في أعمال كل منهما. }
\end{aligned}
$$

\author{
Mohamed Nassim Chikhi \\ Département des lettres et \\ langue française
}

Université des Frères Mentouri Constantine

\begin{abstract}
Introduction :
Lorsque l'on s'interroge sur la relation qui unit Albert Camus et Romain Gary - deux figures littéraires marquantes du siècle précédent - on ne peut qu'adhérer à la célèbre sentence de Voltaire : "les beaux esprits se rencontrent $»$. Ces "jumeaux solaires" que l'admiration et l'amitié liaient, ont en effet en partage, au-delà d'innombrables intuitions communes, au-delà d'un humanisme désabusé et problématique, une même fascination pour le mythe, ainsi que l'ambition de restituer à la littérature sa dimension éminemment existentielle.
\end{abstract}


Le mythe pourrait donc constituer un lieu de rencontre, un axe de lecture, le fil d'Ariane permettant de nous guider et de relier deux œuvres qui peuvent sembler très éloignées l'une de l'autre.

En effet, de prime abord, rien ne semble rapprocher ces écrivains : Camus est le fils de la Méditerranée, Gary celui des neiges et des brumes de Vilnius. Le premier voue un amour sans bornes aux siens et à sa terre natale, le second est un apatride, un métèque, une sorte de Juif errant prônant le cosmopolitisme. Ajoutons à cela le fait que ces tempéraments fortement contrastés n'ont pas manqué de se répercuter sur l'écriture: Albert Camus est considéré, à juste raison, comme le dernier des classiques français, appliquant à la langue l'esprit de clarté typiquement hexagonal. Romain Gary, pour sa part, polyglotte, héritier entre autres des grands ogres russes, excelle dans la subversion du langage, bouleversant la syntaxe, saturant une langue parfois volontairement fautive et faussement naïve de jeux mots et d'anomalies du langage.

Mais en dépit des dissimilitudes les séparant, ces deux écrivains, de la même génération, partagent l'entérinement d'un double constat fondamental : d'une part, la perte définitive du sens et le caractère radicalement absurde de l'existence qui en résulte, de l'autre, la nécessité, voire l'urgence, de convoquer le mythe, qui seul serait en mesure de révéler l'ineffable et de restituer à la littérature la puissance d'une parole fondatrice, perdue dans les tréfonds de la mémoire collective. Ils s'accordent aussi sur l'idée que le constat du désenchantement $d u$ monde ${ }^{(1)}$ ne peut en aucun cas constituer une fin en soi, clôturant définitivement la pensée et plongeant l'homme dans la déréliction. Au contraire, l'acceptation de l'inanité de l'existence est perçue par ces deux romanciers comme l'opportunité fabuleuse d'ouvrir de nouvelles perspectives littéraires, d'étendre à l'infini le champ du possible. Or, seul le mythe à travers l'art romanesque, art existentiel par excellence, est en mesure de permettre la concrétisation de telles aspirations artistiques. La définition minimaliste de Christophe Pérez prend ici tout son sens: "Le mythe est avant tout une production esthétique qui se manifeste dans l'art, et particulièrement dans le roman.» (Pérez, 2009: 223). Il rejoint sur ce point Gilbert Durand qui considère «la littérature, et spécialement [le] récit romanesque» comme «département du mythe ». (Durand, $1961: 12$ )

De là, on perçoit déjà l'ampleur que le mythe va prendre dans les œuvres respectives d'Albert Camus et de Romain Gary. Il va nous falloir approfondir la réflexion sur cette dimension mythique et sur la manière dont elle se manifeste. Mais avant cela, nous devons d'abord nous arrêter un instant sur le problème épineux de la définition du mythe. Certes, les pages de cet article ne pourraient suffire à aborder de manière appropriée une question aussi complexe, mais il nous est impossible d'éviter cet écueil. Rappelons tout d'abord que des décennies de recherches dans des domaines aussi variés que l'anthropologie, l'histoire des religions, la psychanalyse, la critique littéraire, 


\section{Le mythe et le réenchantement de l'écriture, Albert Camus et Romain Gary en quête d'une parole originelle}

etc. ont échoué à donner au terme "mythe" des critères de distinctions irrécusables. Le mythe demeure une notion nébuleuse, fuyante, s'évaporant au moment même où l'on croit la saisir. Il n'est donc pas surprenant que Suzanne Saïd puisse qualifier le mythe de « catégorie poubelle» (Saïd, 1993 : 7), André Siganos de «concept fourre-tout» et que Marcelle Détienne remette en question l'existence même du mythe (Détienne, 1981:162).

Ce scepticisme méprisant, s'il doit impérativement être pris en compte, s'oppose radicalement à l'esprit même de l'étude des mythes en littérature, et plus spécifiquement à la mythocritique. Cette dernière, comme l'indique Pierre Brunel, considère la présence de l'élément mythique dans le texte comme essentiellement signifiante. C'est même à partir de cet élément que doit s'organiser l'analyse du texte ${ }^{(3)}$. Pour esquiver les postures dédaigneuses et produire un discours valide, il faut, comme Charles Delattre, intégrer le caractère polymorphe et l'ambivalence du mythe. ${ }^{(4)}$ Dans cette perspective, Pierre Brunel a retenu la définition de Mircea Eliade qui, selon lui, a le mérite de fournir une acception large et extensive: "Le mythe raconte une histoire sacrée; il relate un événement qui a eu lieu dans le temps primordial, le temps fabuleux des "commencements" 》 (Eliade, 1963 :15). Cette définition met en lumière le caractère narratif du mythe sur lequel s'accordent la plupart des chercheurs. Elle nous amène également à nous interroger sur le rapport qu'entretient l'expression littéraire dans notre monde contemporain, sécularisé, désenchanté, technicisé, et la pré-littérature orale et primitive qui appartient au domaine du mythe? Celle-là même qui octroyait aux hommes premiers à travers un savant mélange de rythmes, de danses, de mots et de symboles, le pouvoir de créer des récits fabuleux, des récits révélant l'ineffable, le secret, le domaine du sacré, et qui permettaient à ces hommes de la horde primitive d'apprivoiser une nature hostile et des phénomènes mystérieux, de justifier l'être, le rite, l'organisation et la hiérarchie sociales, de se soustraire, enfin, au monde profane pour rejoindre un temps devenu réversible, le temps fabuleux des commencements.

On pourrait se demander pour quelle raison la narration littéraire mime-t-elle et s'approprie-elle ce corps étranger qu'est le mythe, ce langage antédiluvien, brutal, fortement contrasté et surchargé symboliquement? Colette Astier estime que le mythe est une narration minimale véhiculant des images maximales. Elle le définit comme "le récit cru de ce qui est cru » ${ }^{(5)}$. C'est-àdire qu'il relate, sans les atténuer, des situations violentes (viol, cannibalisme, inceste, fratricide, infanticide, errance...). En somme, il «dit fortement ce qui en soi est déjà fort $\rangle^{(6)}$. On comprend de ce fait que le mythe puisse offrir à la littérature la puissance d'une parole des origines. Cette puissance archaïque est l'occasion pour l'œuvre littéraire de transgresser ses propres codes, de transcender sa dimension de création individuelle et profane pour s'inscrire dans le mouvement plus vaste de l'inconscient collectif, et ainsi d'incarner 
l'âme d'un peuple, d'une génération, d'une culture... Cependant, il serait faux de ne voir dans le texte littéraire qu'un simple réceptacle conservant et véhiculant des récits mythiques. La littérature peut parfois s'approprier les pouvoirs du mythe ou devenir elle-même créatrice de mythes nouveaux, qu'ils soient tirés du réel ou spécifiquement littéraires (César, Napoléon, Faust, Don Juan, etc.).

Quoi qu'il en soi, nous clorons la question de la définition du mythe en considérant avec Véronique Gely ${ }^{(7)}$ qu'il serait vain de vouloir restreindre le large spectre des définitions du mot "mythe". Autrement dit, de réduire sa vitalité, sa flexibilité, sa multiplicité en le spécifiant conceptuellement, d'autant plus que nous nous intéresserons moins à l'acception du mythe en lui-même qu'aux formes et à la place que ce dernier a pu prendre dans l'entreprise de création littéraire telle que la conçoivent Albert Camus et Romain Gray.

\section{L'opposition mythe/réalité à la source de la création littéraire chez Romain Gary}

Romain Gary estime que le roman offre au créateur l'occasion de se démultiplier, d'expérimenter l'altérité et de vivre à travers ses propres personnages des réalités diverses. L'art romanesque est de ce fait perçu comme le moyen de se soustraire aux limites que nous impose le réel. De là émane cette rivalité constante, voire obsessionnelle, entre imaginaire et réalité irradiant son œuvre entière. Ainsi, il confie :

«Attaqué par le réel sur tous les fronts, refoulé de toutes parts, me heurtant pourtant à mes limites, je pris l'habitude de me réfugier dans un monde imaginaire et à $y$ vivre, à travers les personnages que j'inventais. " (Anissimov, 2004 : 46).

Cet antagonisme entre monde réel et domaine de l'imaginaire constitue justement le lieu privilégié où le mythe va venir prendre place pour se déployer. Si, sous l'action conjuguée des sciences, de l'individualisme et de la solitude $^{(8)}$, la réalité démystifie le monde, le fragmente, limite l'homme aux déterminations spatio-temporelles qui compromettent irrémédiablement son désir d'autonomie, l'imaginaire offre, quant à lui, la possibilité de transcender le réel, d'expérimenter l'infini, l'intemporalité et la liberté absolue. Néanmoins, il faut signaler que cette révolte contre le réel ne s'exprime jamais dans l'œuvre de Romain Gary, comme c'est d'ailleurs le cas pour Camus, par une simple négation, mais davantage par une affirmation mythique. Car comme le fait remarquer Katell Thomas, le mythe, cet absolu à atteindre, ne peut prendre forme qu'à partir de la réalité lorsque celle-ci est transfigurée par l'imagination. On retrouve donc chez Gary, dans le rapport qu'il entretient avec le mythe, un double mouvement auquel Albert Camus aurait sans doute adhéré : Dans un premier temps, il s'agit d'extraire le mythe de la réalité, par la suite, il faut se soustraire soi-même au réel par le mythe et la puissance créatrice de l'écriture. 


\section{Le mythe et le réenchantement de l'écriture, Albert Camus et Romain Gary en quête d'une parole originelle}

Dans un essai intitulé Pour Sganarelle, Romain Gary confie la détresse et le sentiment de déperdition qui l'assaillent dans le moment précédant la création littéraire :

«Je connais depuis longtemps ces états négatifs de la conscience, cette absence de quelque chose ou de quelqu'un, et ce remords qui me gagne dès que je laisse la réalité s'accumuler autour de moi comme un matériau refusé. (...). La Puissance de la réalité me soumet (...) Je n'existe plus : je me dissous. (...). Mutilé, infirme, incomplet, un poisson hors de l'eau, un romancier sans roman. Au lieu de tirer l'œuvre des limbes, ce sont les limbes qui me tirent, me gagnent, et me font perdre mes contours, le sentiment de ma propre réalité » (Gary, 2003 : 11).

Un peu plus loin, Romain Gary définit ce qu'il attend de l'expérience créatrice plénière: elle doit lui permettre de «rivaliser avec la puissance, celle du monde authentique. ». Elle doit rendre possible sa volonté " de [se] diversifier par de nouvelles et multiples identités et de vivre à travers elles une expérience totale ». La littérature est ainsi censée l'aider à se libérer de "l'habitude et de la claustrophobie, d'un état individuel, de [son] petit Royaume du Je ». Enfin, la création littéraire constitue " un besoin de jouir de la vie jusqu'à possession totale, d'être tout.» (Gary, $2003: 12)$.

Pour Romain Gary l'expérience créatrice offre donc l'occasion d'une transmutation ontologique de l'écrivain. Le roman devient un lieu de transition de la condition de "profane » à ce qu'il qualifie de "vie authentique ». On passe, dans les extraits précédents, de la fragmentation à l'unité, de la détresse à l'allégresse, de la déperdition à la toute puissance, de l'effacement à l'affirmation. Le "je", ce bagne existentiel, ce moi-prison est transcendé par un moi-évasion, plus précisément par ce que Gary qualifie d'«aspiration à la collectivité, à la totalité ». Cette aspiration, qui revient de manière obsédante dans l'œuvre de Gary, peut faire penser au célèbre cogito camusien : "je me révolte donc nous sommes ». Il y a là sans doute entre Gary et Camus une intuition commune : le passage du "je" au "nous" par la révolte.

Il est par ailleurs tout à fait remarquable de deviner dans l'itinéraire créateur, tel qu'il est décrit par Romain Gary, une sorte de rite initiatique, équivalant à celui que l'on retrouve dans les récits mythiques. Ce cheminement a été qualifié de " comportement mythico-poétique» par Max Bilen. Il le définit comme un état à la faveur duquel l'artiste «tente d'accéder, par les voies de l'imaginaire, à une métamorphose de statut qui lui permettrait de s'affranchir de toute détermination et de vivre dans un monde devenu réversible.» ${ }^{(9)}$. Cette initiation, dont on peut retrouver la trace aussi bien dans les récits mythiques anciens que dans le roman contemporain, se déroule, selon Max Bilen, en trois phases : ségrégation, révélation, métamorphose. Romain Gary, à travers le cheminement de la création qu'il décrit: (négativité, nouvelle naissance, transmutation ontologique), nous permet de déduire que, chez cet écrivain, le 
rôle du héros mythique n'est plus seulement endossé par le personnage romanesque, mais par l'auteur lui-même, l'homme en chair et en os qui assume la condition mythique de l'initiation et la projette sur ses personnages.

La dimension mythique de la création littéraire nous offre de ce fait une autre grille de lecture concernant l'affaire Émile Ajar: au-delà de la simple supercherie littéraire, du mauvais tour joué à la critique que l'on a bien voulu y voir, cette mystification constitue une mise en pratique de la théorie du roman total exposée par Romain Gary dans son essai Pour Sganarelle. Elle pourrait éventuellement être comprise comme une tentative donquichottesque de se métamorphoser en mythe vivant, de faire surgir le mythe dans le monde profane par l'élimination de ce qui sépare la réalité de sa représentation dans le roman. Le roman, on l'aura compris, devient un lieu de transgression des frontières, un espace de jonction entre imaginaire et réalité annonçant ce que le narrateur de Gros-Câlin qualifie de «fin de l'impossible ».

On effleure ici, dans cette révolte contre le réel, dans l'attente désabusée d'une fin de l'impossible ce qui sépare peut-être le plus intimement Romain Gary d'Albert Camus. L'auteur de L'Etranger considère en effet que la pensée d'un homme est avant tout l'expression d'une nostalgie, une nostalgie d'innocence. Justement, le mouvement mythique chez Gary est contraire à celui de Camus, au lieu de la pente régressive et nostalgique, du retour en arrière camusien qui nous ramène au paradis perdu, à Tipaza, aux sources secrètes et mythiques de l'œuvre, il serait plus approprié de parler à propos de Gary d'une projection en avant, d'une sorte de "messianisme" qu'il a lui-même revendiqué et thématisé à maintes reprises dans son œuvre. Ces personnages sont souvent dans l'attente d'un évènement avenir, d'un messie, d'une promesse. Toutefois cette aspiration est confortée à cet impossible - qu'Albert Camus appelle "l'absurde" - et auquel Gary a tenté d'échapper en multipliant les vies, les identités et les modes d'écriture.

\section{Le mythe comme amplificateur du réel chez Albert Camus}

L'œuvre d'Albert Camus se caractérise elle aussi par un foisonnement mythique inouï au point que Jean Sarocchi préfère parler d'une mythologie camusienne plutôt que d'une philosophie. Camus déclare lui-même: "je ne suis pas un romancier au sens où on l'entend, mais plutôt un artiste qui crée des mythes » (Todd, 2012: 701). L'auteur du Mythe de Sisyphe est-il pour autant un créateur de mythes? Si Goëten Picon lui accorde le fait «d'avoir donné de l'homme contemporain la seule expression mythique qu'il ait eu jusqu'à présent» (Sarocchi, 1968: 17), d'autres, à l'instar de Rachelle Bespaloff, lui dénient ce pouvoir. Quoi qu'il en soit, Camus considère que l'une des tâches principales de l'écrivain, ou du philosophe artiste, à l'image de Nietzsche, est de prendre en charge les mythes, de leur insuffler la vie, car, affirme-il, ceux-ci ne peuvent exister en dehors de leurs incarnations successives : «Les mythes n'ont point de vie par eux-mêmes. Ils attendent que 


\section{Le mythe et le réenchantement de l'écriture, Albert Camus et Romain \\ Gary en quête d'une parole originelle}

nous les incarnions. Qu'un seul homme au monde réponde à leur appel, et ils nous offrent leur sève intacte » (Camus, 1954 : 123).

Albert Camus va donc entreprendre à travers son œuvre, aussi bien philosophique que littéraire, ce que l'on pourrait qualifier de "réhabilitions du muthos". Il estime, dans Le mythe de Sisyphe que le roman possède sa propre « logique, ses raisonnements, son intuition et ses postulats 》 (Camus, Le mythe de Sisyphe, $2011:$ 137) et que l'art romanesque permet d'explorer des vérités profondes qui inévitablement mentiraient si on les désincarnait dans un discours cohérent. Seuls, "les symboles et les mythes les préservent telles des pièces d'une énigme que l'on ne résout pas, mais que l'on joue» (Sarocchi, $1968: 16)$.

Dans cette perspective, Camus assure qu' «incapable de sublimer le réel, la pensée s'arrête à le mimer ». Par mimer, il entend le fait d'accroître sensiblement l'intensité du réel. Sublimer, en revanche, consisterait à «superposer un système explicatif» (Sarocchi, 1968: 15). Or, toute explication, qui se proposerait de synthétiser un monde radicalement absurde, est inéluctablement vouée à l'échec. Seul le mythe - ce corps étranger que le roman annexe et s'approprie -, ne saurait mentir étant donné qu'il n'a pas la prétention de réduire la réalité, mais d'augmenter considérablement son intensité.

Emergence des mythes dans les ouvres d'Albert Camus et de Romain Gary

Tout le long de son œuvre, Albert Camus va donc exploiter l'ironie, le paradoxe, les symboles, ressusciter des figures mythiques et archaïques afin de parler de l'être et du monde, d'exprimer pleinement la condition humaine. Jean Sarocchi estime à juste titre que Camus parvient à incarner parfaitement le sentiment de l'absurde avec Meursault - une sorte de Sisyphe moderne. Il exprime par ailleurs la révolte de son siècle à travers Prométhée ; il renouvelle les thèmes de l'exil et de la nostalgie en ressuscitant Ulysse regagnant Ithaque dans Retour à Tipaza; il annonce le mythe de Némésis pour traduire son appétit de vivre ${ }^{(10)}$; il actualise la malédiction d'Edipe, les mythèmes du fratricide et de l'errance caïniques ${ }^{(11)}$ dans le Malentendu; enfin, la beauté d'Hélène, le donjuanisme étendu à la nature et le mythe du Minotaure dans Noces... À la fin de sa vie, il eut pour projet de monter une pièce qui aurait abordé la révolte faustienne ${ }^{(12)}$, mais une mort précoce l'en empêcha. Gary pour sa part actualisa ce mythe dans son roman Les mangeurs d'étoiles avec un certain parti pris de démystification.

Pour ce qui est de Romain Gary, le mythe central de son œuvre, celui qui revient de manière obsédante dans plusieurs de ses romans - notamment Europa, Le Grand Vestiaire, La danse de Gengis Cohn, Pseudo, Gros-Câlin, etc. - semble être celui du double, du Doppelgänger ${ }^{(13)}$, qu'il a revendiqué en 
tant que tel et thématisé sous toutes ses formes: picaresque, donjuanesque, faustienne, donquichottesque... Dans Le Grand Vestiaire, Gary proclame l'obsolescence du " je", instance restrictive, chargée d'un passé douloureux, en écrivant cette phrase étrange : "Je me suis toujours été un autre (Gary, 2004 : 142), qui n'est pas sans rappeler le «je est un autre » de Rimbaud ou «el otro» de Borges. Camus également ouvre la voie à l'évasion du moiprison, mais d'une toute autre manière : en revendiquant d'abord, une sorte de noce païenne et lyrique avec le monde : «Et ouvertement je vouai mon cœur à la terre (...) je me liai à elle d'un lien mortel $»^{(14)}$, et plus tard, durant la période de l'Occupation, en appelant au passage du "je" au "nous" sur le chemin de la révolte.

Mais c'est dans l'œuvre de Romain Gary et particulièrement dans Pseudo signé Émile Ajar - que la négation du sujet de l'énonciation atteint son comble. Cette négation a pour conséquence l'effacement de l'identité de l'auteur luimême. Le lecteur ne parvient plus à distinguer la fiction du réel, le vrai du faux, le double de l'authentique, le personnage narrateur de l'auteur... Cette obsession de devenir un autre, de se dédoubler, de brouiller les pistes pourrait dévoiler un mécanisme de défense, une sorte d'artifice pour se dissimuler à la manière de son animal fétiche, le Caméléon. Elle peut également être interprétée comme l'expression d'une révolte contre la finitude humaine, contre tout ce qui restreint le champ du possible. Car finalement, à travers le mythe du double, et grâce à la littérature, Gary s'empare d'un pouvoir divin, celui de se métamorphoser, de nier les déterminations spatio-temporelles, de renaître pour vivre dans un monde devenu réversible.

On pourrait donc déduire spontanément que le lieu d'intersection où les mythologies camusienne et garyenne se coupent réciproquement se situerait auprès des mythes de la révolte. Ceux-ci incarnent essentiellement la rébellion désespérée de l'homme contre des puissances infiniment supérieures: Prométhée «martyr éternel exclu à jamais du pardon » (Camus, 2011: 45), Antigone et sa "révolte réactionnaire» (Camus, 2011: 47), Faust et le " mensonge rassurant de Goethe » (Gary, 1981 : 264), Don Juan, Caïn, etc. Si ces figures de la révolte, qui connurent leur zénith à l'époque romantique, semblent s'essouffler au cours du $\mathrm{XX}^{\mathrm{e}}$ siècle, il n'en demeure pas moins que Pierre Albouy ${ }^{(15)}$, reprenant le titre d'un essai de Camus, n'hésite pas à qualifier cette période de siècle de l'Homme révolté. Toutefois, il ne tarde pas à nuancer son propos : la révolte, triomphante durant la période romantique, s'achève désormais dans l'échec et la désillusion. On peut le constater par exemple dans le roman d'André Malraux où tous les révolutionnaires échouent. Il en va de même chez Albert Camus et Romain Gary: L'Etranger et Les mangeurs d'étoiles, pour ne citer que ces romans, expriment parfaitement la désillusion qui caractérise la seconde partie du siècle précédent. 


\section{Le mythe et le réenchantement de l'écriture, Albert Camus et Romain \\ Gary en quête d'une parole originelle}

Le $\mathrm{XX}^{\mathrm{e}}$ siècle fut en effet la période de l'histoire où le désenchantement $d u$ monde, entrepris plusieurs siècles auparavant par les penseurs européens, allait atteindre véritablement son point d'achèvement : un siècle de larmes, de fer, de feu et de barbarie. Albert Camus et Romain Gary avaient perçu que la démystification à outrance ne fut en réalité que l'envers d'un messianisme conquérant porteur d'utopies meurtrières. Autant dire que l'élucidation des mystères du monde n'a jamais été en mesure d'étancher la soif d'absolu qui caractérise homo sapiens, cette espèce fabulatrice, cet animal éminemment mythologique. Ils ont proposé une conception laïque du mythe en l'intégrant dans leur entreprise de création. Et à travers la littérature, ils ont tenté de "remythifier" le monde, de le "réenchanter" tout en assumant les vicissitudes de l'ici-bas aussi bien dans la souffrance que dans les moments de grâce. Ils offrent, de ce fait, au lecteur du XXI siècle une expression mythique puissante et peut-être même, osons le mot, l'ébauche d'une spiritualité.

\section{Notes:}

1- "Le désenchantement du monde" est une expression attribuée au sociologue allemand Max Weber. Elle renvoie à la sécularisation du monde occidental, à la perte du sens, au déclin des valeurs et au triomphe du nihilisme.

2- A. Siganos, « Définition du mythe », Questions de mythocritique, p.93.

3- P. Brunel, Mythocritique : Théorie et parcours. Paris, PUF, Coll.

«Ecriture », 1992, p.82.

4- C. Delattre, Manuel de mythologie grecque, Paris, Bréal, 2005, pp. 232-233.

5-C. Astier, « Interférences et coïncidences des narrations littéraire et mythologique », dans Le Dictionnaire des mythes littéraires, (sous la direction de P. Brunel), Monaco, éd. du Rocher, 2003, p. 1079.

6- Ibid.

7- V. Gely, « Pour une Mythopoétique : quelques propositions sur les rapports entre mythe et fiction ", Vox Poetica, mai 2006, URL : http://www.vox-

poetica.org/sflgc/biblio/gely.html

8-K. Thomas, « Romain Gary songe humain », Acta fabula, vol. 11, $\mathrm{n}^{\circ}$ 5, Notes de lecture, mai 2010, URL: http://www.fabula.org/revue/document5701.php 9-M. Bilen, " Comportement mythico-poétique », dans Le Dictionnaire des mythes littéraires, op.cit., p. 357.

10- O. Todd, Albert Camus, une vie, op.cit., p.969.

11-Pour ce qui est du mythe de Caïn dans l'œuvre de Camus, voir l'article de C. Hussherr, « Lecture du mythe de Caïn et Abel chez Albert Camus et Pierre Emmanuel, de l'exil terrestre à l'enracinement dans la terre. », Presses universitaires de Paris Ouest, 2009, URL :

http://books.openedition.org/pupo/1447?lang=fr

12- O. Todd, Albert Camus, une vie, op.cit., p.970. 
13- “Doppelgänger', terme forgé par Jean-Paul Richter, en 1796, et qui signifie littéralement : " celui qui marche à côté, le compagnon de route ». 14- Epigraphe de L'Homme révolté, Paris, Gallimard, coll. « Folio Essais », 2011.

15-P. Albouy, Mythes et mythologies dans la littérature française, Paris, Armand Colin, 1998, p. 97.

Bibliographie :

Albouy, P., Mythes et mythologies dans la littérature française, Paris : Armand Colin, 1998.

Anissimov, M., Romain Gary, le Caméléon, Paris, Editions Denoël, 2004.

Brunel, P., Le Dictionnaire des mythes littéraires, Monaco, éd. du Rocher, 2003.

Brunel, P., Mythocritique : Théorie et parcours, Paris, PUF, 1992.

Camus, A., L'Homme révolté, Paris, Gallimard, 2011.

Camus, A., Le mythe de Sisyphe, Paris Gallimard, 2011.

Camus, A., Prométhée aux Enfers, dans L'Eté. Paris, Gallimard, 1954.

Détienne, M., L'invention de la mythologie, Paris, Gallimard, 1981.

Eliade, M., Aspect du mythe, Paris, Gallimard, 1963.

Gary, R., Le Grand Vestiaire, Paris, Gallimard, 2004.

Gary, R., Les mangeurs d'étoiles, Paris, Gallimard, 1981.

Gary, R., Pour Sganarelle, Recherche d'un personnage et d'un roman, Paris, Gallimard, 2003

Gilbert, D., Le Décor mythique de «La Chartreuse de Parme », Paris, José Corti, 1961.

Pérez, C., Romain Gary, la Comédie de l'Absolu, Bordeaux, Eurédit, 2009.

Saïd, S., Approche de la mythologie grecque, Paris, Nathan, 1993.

Sarocchi, J., Camus, Paris, Presse Universitaire de France, 1968.

Todd, O., Albert Camus, une vie, Paris, Gallimard, 2012. 